\title{
Similarity-Based Appearance-Prior for Fitting a Subdivision Mesh in Gene Expression Images
}

\author{
Yen H. Le ${ }^{1}$, Uday Kurkure ${ }^{1}$, Nikos Paragios ${ }^{1,2}$, \\ Tao $\mathrm{Ju}^{3}$, James P. Carson ${ }^{4}$, and Ioannis A. Kakadiaris ${ }^{1}$ \\ 1 Computational Biomedicine Lab, University of Houston, Houston, TX, USA \\ 2 Center for Visual Computing, Ecole Centrale de Paris, France \\ 3 Washington University in St. Louis, MO, USA \\ 4 Pacific Northwest National Laboratory, Richland, WA, USA
}

\begin{abstract}
Automated segmentation of multi-part anatomical objects in images is a challenging task. In this paper, we propose a similarity-based appearance-prior to fit a compartmental geometric atlas of the mouse brain in gene expression images. A subdivision mesh which is used to model the geometry is deformed using a Markov Random Field (MRF) framework. The proposed appearance-prior is computed as a function of the similarity between local patches at corresponding atlas locations from two images. In addition, we introduce a similarity-saliency score to select the mesh points that are relevant for the computation of the proposed prior. Our method significantly improves the accuracy of the atlas fitting, especially in the regions that are influenced by the selected similarity-salient points, and outperforms the previous subdivision mesh fitting methods for gene expression images.
\end{abstract}

Keywords: segmentation, gene expression image, subdivision mesh.

\section{Introduction}

Automated segmentation of the anatomical objects in images is a difficult task. It is even more challenging to segment multiple neighboring objects and/or multipart objects. The use of appropriate and effective image appearance models plays a critical role in such segmentation tasks. Most of the existing approaches rely on the assumptions that the regions of interest are distinct (e.g., heart ventricles in MRI and contrast CT images) and have similar intensity patterns at particular locations (e.g., white and gray matter in the brain MRI images) across multiple images (images acquired from different subjects or the same subject at different time instances). However, these assumptions do not hold for gene expression images because of their complex appearance. The intensity at a pixel is related to the amount of precipitate in a cell, unlike in CT/MRI images, where it represents a particular tissue type. Therefore, the gene expression images provide incomplete anatomical information. Thus, the incorporation of appearance-prior in a segmentation method is non-trivial for gene expression images. 
Geometric model-to-image registration-based methods use soft shape constraints that are inherent to the model geometry which conforms with the object of interest. Various deformable model representations (e.g., simplex mesh 1], m-rep [2], subdivision mesh [3]) have been used to characterize the complex geometry of the structures. Of notable interest is the subdivision mesh-based representation that can compactly represent the complex, multi-part structures in a hierarchical manner using very few control points. A subdivision mesh-based geometric atlas has been used to segment the multi-part, mouse brain structures in the gene expression images [4 7]. Among those methods, an appearance-prior is employed weakly as a segmented brain boundary [4 7], anatomical landmarks [5, 6], or a texture of internal sub-region boundaries [5, 6]. Image-to-image registration-based approaches [8 11] use the deformation field to map the predefined segmentation labels from the reference image/atlas to the given image. However, these approaches require image warping at each iteration and can lead to nonphysical deformations to satisfy the energy constraints while recovering large deformations.

In this paper, we propose a novel approach for fitting the internal regions of a subdivision-based geometric atlas to anatomical structures. Our main contributions are: (i) we introduce a novel similarity-based appearance-prior for geometric atlas fitting in gene expression images, and (ii) we present a systematic method based on the modified similarity-saliency to identify the key vertices of the geometric atlas to be used to compute the appearance-prior during the deformation. Specifically, we modified the method proposed by Kurkure et al. 7] for model-to-image fitting of the subdivision atlas to include the proposed similarity-based appearance-prior. The main difference is that Kurkure et al. 7] did not include any appearance-prior as part of the framework, whereas our proposed prior enables fitting the geometric model to the internal regions of the brain (the main limitation of [7]). This is particularly important for this application where an accurate correspondence across gene expression images is needed to build a spatial database which can be queried for similarities in gene expression patterns to find potential interactive relationships between different genes in the same anatomical sub-region. Our proposed similarity-based appearanceprior is computed as a function of similarity of local patches at corresponding atlas locations between two images. Moreover, it can be used to fit other geometric models as a part of the energy/cost function (e.g., b-spline curve fitting on a vessel). Though we used mutual information as the similarity metric, it can be replaced with any suitable similarity measure depending on the properties of the images.

\section{Background}

Our segmentation method employs a Markov random field-based subdivision mesh fitting framework proposed by Kurkure et al. [7]. In that framework, the segmentation is performed by fitting a hybrid geometrical-anatomical atlas to the input image. The atlas is modeled by a subdivision mesh [4]. For any given 
input image, the fitting process deforms the subdivision mesh atlas based on the internal energy (of the mesh) and external energy (of the image), which includes energy build from our proposed similarity-based appearance-prior.

The subdivision mesh [4] is organized in a multi-resolution fashion, where the geometric mesh at the $k^{t h}$ level is denoted by $M^{k}$. The mesh vertices at the coarsest level $M^{0}$ are referred to as control points whose locations completely determine the locations of the mesh points at higher resolution. The position of a mesh vertex $i$ at the level $k$ as $M^{k}(i)$ is computed as a linear combination of positions of the control points as, $M^{k}(i)=\sum_{j} \eta_{j}^{k}(i) M^{0}(j)$, where $\eta_{j}^{k}(i)$ denotes the influence of the control point $M^{0}(j)$ to the mesh vertex $M^{k}(i)$. The optimal fitting of the subdivision mesh is determined by estimating the optimal locations of the control points on the image.

The estimation of the optimal locations of the control points is formulated as a discrete label assignment problem, where the labels denote the discretized displacements of the control points. This label assignment problem is solved using MRF where an energy function is minimized. The energy function includes boundary-based (shape-based) energy, landmark-based energy, label regularization energy, and internal energy [7]. The label regularization and internal energy terms provide local geometric constraints, whereas the boundary-based (shapebased) and landmark-based energy terms provide global geometric constraints. Therefore, we categorize these energy terms as geometric priors, $E^{G}(f \mid I)$, where $f$ denotes the labels of the displacements of the control points and $I$ denotes the input image.

\section{Similarity-Based Appearance-Prior}

In this section, we provide details of our geometric atlas fitting approach. First, we introduce the proposed similarity-based appearance-prior. Then, we present the modified similarity-saliency measure and explain how it is used to select relevant appearance points. Finally, we describe the integration of the proposed appearance-prior in the MRF fitting framework [7].

\subsection{Energy Function}

Our appearance energy function is computed as the sum of similarities on selected pairs of appearance points from a reference image and the input image. Let $R$ be the reference image, $I$ be the input image, and $\mathcal{S}$ be the set of coordinates of appearance points, the appearance energy function $E^{A}$ can be computed as: $E^{A}(I)=\sum_{s \in \mathcal{S}} \psi(R(s), I(s))$, where $\psi(p, q)$ denotes the similarity metric between two image patches centered at point $p$ and point $q$. In this paper, we use normalized mutual information (NMI) [12] as the similarity metric. The set of appearance points is determined by examining the pattern of the similaritysaliency on the set of training images.

Similarity-Saliency: Given two images $I_{1}$ and $I_{2}$, an image point $u$ in $I_{1}$ is similarity-salient with respect to the image point $v$ in $I_{2}$ if $v$ exhibits higher 
similarity, than its neighbors, to $u$. The concept of this similarity-saliency is similar to the mutual-saliency used in [13] to perform Gabor feature selection and to weigh the similarity at each point to determine the corresponding points. However, we use the similarity-saliency to determine which mesh points are more reliable to use for the computation of the appearance-prior during mesh fitting. To compute the similarity-saliency, we define three types of regions in the neighborhood of the point $v$ in the given image: (i) core neighborhood $\left(\mathcal{N}_{C}\right)$, (ii) transitional neighborhood $\left(\mathcal{N}_{T}\right.$, which is ignored in the computation because of smooth transition in degree of similarity), and (iii) peripheral neighborhood $\left(\mathcal{N}_{P}\right)$ as illustrated in the left panel of Fig. 11. The similarity-saliency $\Upsilon(u, v)$ is then computed as: $\Upsilon(u, v)=\frac{\frac{1}{\frac{\mathcal{N}_{C}(v) \mid}{1} \sum_{m \in \mathcal{N}_{C}(v)} \psi(u, m)}}{\frac{1}{\left|\mathcal{N}_{P}(v)\right|} \sum_{n \in \mathcal{N}_{P}(v)} \psi(u, n)}$.

Appearance Points Selection: In the gene expression images, all the genes typically do not express at the same level. Therefore, the gene expression images provide incomplete anatomical information. This issue leads to the weak similarity-saliency between some corresponding regions even when the two images have been properly aligned. In order to improve the robustness of the energy estimation, we select only a subset of all image points to compute the appearance energy function (the appearance points). The appearance points are determined from the pattern of similarity-saliency on a set of training images against the Nissl-stained image (NSI), which is also the template image used to fit test images. Since the NSI is generated using a universal gene probe to which all the cells in a tissue sample respond, it has maximum anatomical information and it exhibits the highest similarity to other gene expression images. Therefore, it is reasonable to introduce a MI-based similarity prior for mesh fitting in the gene expression images when using the NSI as the reference image. For each image in the training set, we manually align the subdivision mesh [4] to achieve point-to-
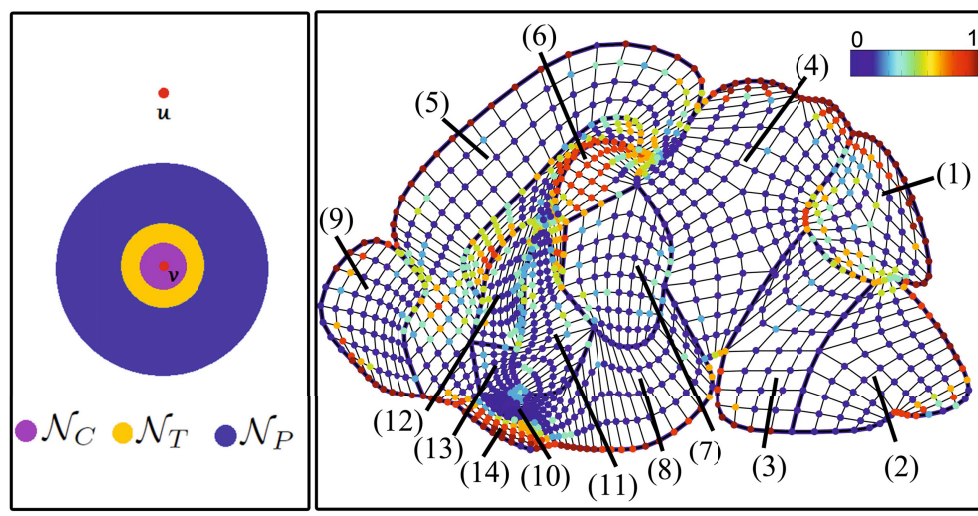

1 Anatomical Regions
(1) Cerebellum
(2) Medulla
(3) Pons
(4) Midbrain
(5) cortex
(6) hippocampus
(7) thalamus
(8) hypothalamus
(9) olfactory bulb
(10) basal forebrain
(11) septum
$(12)$ striatum
$(13,14)$ ventral
striatum

Fig. 1. Left: Illustration of mutual saliency definition for points $v$ in image $I_{2}$ with respect to point $u$ in image $I_{1}$. Right: A subdivision mesh, at subdivision 2, overlaid by average similarity-saliency scores of mesh vertices after scaling for visualization. 
point correspondence across all training images. Then, we compute the average similarity-saliency score for each point $u$ in the reference image (the NSI) by taking the average of the similarity-saliency between $u$ and its corresponding points over all training images. For each point $u$, a high average similarity-saliency score expresses the high consistency of being a similarity salient point across most of the training images, and thus, it should be selected as an appearance point. The right panel of Fig. 1 depicts the color coded map of the average similaritysaliency score on the reference image. Note that most similarity-salient points are observed near the brain boundary, the hippocampus, the ventral striatum and the basal forebrain. Finally, we select similarity-salient points which have the average similarity-saliency score above a given threshold to be used as the appearance points.

\subsection{Markov Random Field with an Appearance Energy Function}

We integrate the proposed appearance energy function into the MRF-based subdivision mesh fitting framework proposed by [7]. In that framework, the fitting problem is modeled as a discrete labeling problem. From the subdivision mesh of the template image, a graph $\mathcal{G}=(\mathcal{V}, \mathcal{E})$ is created, where the set of $N$ nodes $\mathcal{V}$ corresponds to the set of mesh control points $M^{0}$. A fitting solution of an input image to the template image is then represented as a configuration $f=\left\{f_{1}, f_{2}, \ldots, f_{N}\right\}$, where $f_{i}$ is the discrete label of the displacements for node $i$. The configuration is then found by minimizing the total energy $E(f \mid I)$, which is the sum of the geometric energy $E^{G}(f \mid I)$ and the appearance energy $E^{A}(f \mid I)$ as follows: $E(f \mid I)=E^{G}(f \mid I)+E^{A}(f \mid I)=\sum_{c \in C} V_{C}^{G}(f \mid I)+\sum_{c \in C} V_{C}^{A}(f \mid I)$. The geometric energy $E^{G}(f \mid I)$ is the sum of clique potentials $V_{C}^{G}(f \mid I)$ over the set of all possible cliques $C$ as described in [7]. The appearance energy $E^{A}(f \mid I)$ is derived from our appearance energy function presented in Section 3.1 . Within the MRF framework, $E^{A}(f \mid I)$ is the sum of clique potentials $V_{C}^{A}(f \mid I)$, which is defined as the sum of pairwise potentials: $E^{A}(f \mid I)=\sum_{\{i, j\} \in \mathcal{E}} V_{i j}^{A}\left(f_{i}, f_{j}\right)$.

The set of graph edges $\mathcal{E}$ contains all the pairs of control points $\{i, j\}$ such that $i$ and $j$ influence the position of at least one common point in the image. For each pair $\{i, j\}$ with the corresponding label $\left(f_{i}, f_{j}\right)$, its clique potential $V_{i j}^{A}\left(f_{i}, f_{j}\right)$ is computed as:

$$
V_{i j}^{A}\left(f_{i}, f_{j}\right)=\sum_{M^{k}(m) \in \mathcal{S}} \hat{\eta}_{i j} \hat{\psi}\left(R\left(M^{k}(m)\right), I\left(\hat{M}_{f_{i}, f_{j}}^{k}(m)\right)\right),
$$

where $\hat{\psi}(p, q)=2-\psi(p, q)$ denotes the dissimilarity between two image patches centered at point $p$ and point $q$. Here, we consider only image points that belong to both appearance points set and mesh vertices set at level $k$. By deforming the mesh template, the point $M^{k}(m)$ on the reference image is mapped to the point $\hat{M}_{f_{i}, f_{j}}^{k}(m)$ on the input image, which can be computed using the subdivision-basis coefficients as: $\hat{M}_{f_{i}, f_{j}}^{k}(m)=M^{k}(m)+\eta_{i}(m) d_{f_{i}}+\eta_{j}(m) d_{f_{j}}$, where $d_{f_{i}}$ and $d_{f_{j}}$ denote the discrete displacements with labels $f_{i}$ and $f_{j}$ respectively. The inverse weight coefficient, $\hat{\eta}_{i j}$ measures the influence of a mesh 


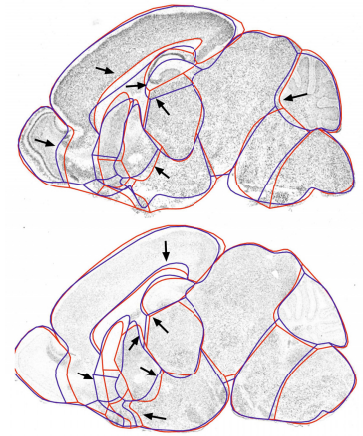

Bello et al.

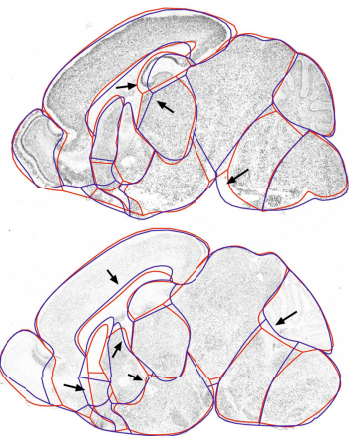

Kurkure et al.
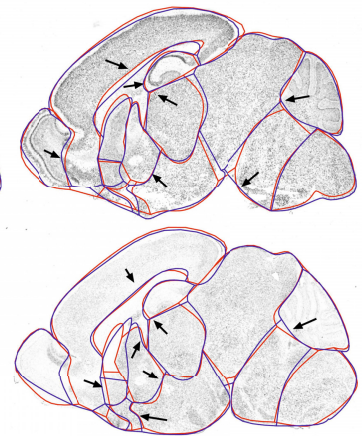

Our Method

Fig. 2. The manually annotated boundaries (red) and the resulting boundaries (blue) of automatic segmentation on images of genes Chrnb4 (top) and Dscr3 (bottom). The arrows indicate the boundaries where our method achieved better alignment than the rest.

vertex $m$ on the pairwise energy of control points $i$ and $j$, and it is given by: $\hat{\eta}_{i j}(m)=\frac{\eta_{i}(m)+\eta_{j}(m)}{\sum_{a, b \in \mathcal{E}}\left(\eta_{a}(m)+\eta_{b}(m)\right)}$. Note that, although the MRF graph is defined by the mesh at the coarsest level $\left(M^{0}\right)$, each configuration is evaluated based on the finer mesh at a higher subdivision level $\left(M^{k}\right)$. This helps to reduce the size of the graph, enabling computation of the priors at a higher resolution. Also, the deformed meshes do not have non-smooth areas, thanks to the label regularization energy and the mesh internal energy.

\section{Results}

We evaluated our method on 100 gene expression images that depict sagittal sections of postnatal day 7 mouse brains at standard section 9 [6, 14]. Each image is $600 \times 1000$ pixels and is rigidly aligned to a reference Nissl-stained image. From the 100 test images, 40 images were included in the training set. The subdivision mesh has 93 control points. From 1,245 mesh points (at subidivision level $k=2$ ), we manually selected 20 appearance points based on the similaritysaliency. Preference was given to the points on the internal boundaries. The radii for $\mathcal{N}_{C}, \mathcal{N}_{T}$ and $\mathcal{N}_{P}$ were set to 7,3 and 5 pixels, respectively. The patch size to compute the mutual information was set to $41 \times 41$ pixels. Note that only a small number of points in the interior regions and in the regional boundaries have high scores (Fig. 1). This also demonstrates the lack of reliable anatomical information in the gene expression images. We did not include any points from the outer boundary as they are already taken into account in the boundary prior. We compare our method with the methods of Bello et al. [6] and Kurkure et al. 7], which also deform a subdivision mesh on the test images. The parameters of the competitive methods were selected as suggested by the original authors. 


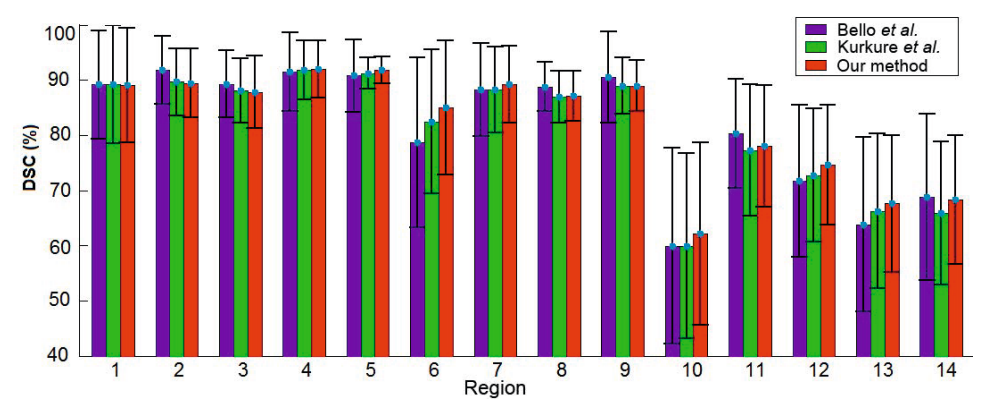

Fig. 3. A quantitative comparison of the mean and standard deviation of the DSC computed from the results of Bello et al. [6], Kurkure et al. [7], and our method

Figure 2 qualitatively indicates that our method results in better alignment of internal boundaries than [6] and [7]. The difference is mostly exhibited in regions 5-7, 10 and 14. For quantitative evaluation, we computed the region overlap ratio with respect to manual annotation using the Dice similarity coefficient (DSC). We plot the mean and standard deviation values of the DSC for all 14 regions in Fig. 3. At significance level of 0.05, our proposed appearance-prior improves the subdivision mesh fitting in seven regions: 5-7, 10 and 12-14 when compared to [7]. In regions that are highly influenced by the selected similarity-salient points $(6,10$ and 14), the difference is remarkable since the mean DSC was improved by $3 \%$ using our method while the standard deviation was reduced. Our method improved the mesh alignment in the neighboring regions $(5,7,11-13)$ as well because of the regularization. For the remaining regions, the results are comparable as they are not influenced by the selected similarity-salient points, hence the mesh in these regions is driven by the other priors. In comparison with Bello's [6] mesh fitting method, our method has higher mean DSC in regions 5-7 and 10-13. In the remaining regions, the mean DSCs from our method are comparable. However, our method has significantly lower standard deviation. This indicates that our method is more robust to the variations in the gene expression images.

\section{Conclusions 1}

In this paper, we have proposed a similarity-based appearance-prior for fitting a geometric mesh model to a multi-part, structure of interest in the gene expression images. It has been incorporated into the Markov Random Field-based subdivision mesh fitting framework of [7]. We have also introduced a similaritysaliency score to select relevant mesh vertices appropriate to define the proposed

\footnotetext{
${ }^{1}$ This work was supported in part by NSF DBI0743691, NIH 1R21NS058553 and by the University of Houston (UH) Eckhard Pfeiffer Endowment Fund. All statements of fact, opinion, or conclusions contained herein are those of the authors and should not be construed as representing the official views or policies of NSF, NIH, or UH.
} 
appearance-prior. Through experimental evaluation, we demonstrate that such a similarity-based appearance-prior is appropriate for gene expression images.

\section{References}

1. Delingette, H.: General object reconstruction based on simplex meshes. International Journal of Computer Vision 32(2), 111-146 (1999)

2. Pizer, S.M., Fletcher, P.T., Joshi, S., Thall, A., Chen, J.Z., Fridman, Y., Fritsch, D.S., Gash, A.G., Glotzer, J.M., Jiroutek, M.R., Lu, C., Muller, K.E., Tracton, G., Yushkevich, P., Chaney, E.L.: Deformable m-reps for 3D medical image segmentation. Int. J. Comput. Vision (IJCV) 55(2-3), 85-106 (2003)

3. Warren, J., Weimer, H.: Subdivision methods for geometric design: A constructive approach. Morgan Kaufmann (2001)

4. Ju, T., Warren, J., Eichele, G., Thaller, C., Chiu, W., Carson, J.: A geometric database for gene expression data. In: Proc. Eurographics Symposium on Geometry Processing, pp. 166-176 (July 2003)

5. Kakadiaris, I.A., Bello, M., Arunachalam, S., Kang, W., Ju, T., Warren, J., Carson, J., Chiu, W., Thaller, C., Eichele, G.: Landmark-Driven, Atlas-Based Segmentation of Mouse Brain Tissue Images Containing Gene Expression Data. In: Barillot, C., Haynor, D.R., Hellier, P. (eds.) MICCAI 2004, Part I. LNCS, vol. 3216, pp. 192-199. Springer, Heidelberg (2004)

6. Bello, M., Ju, T., Carson, J.P., Warren, J., Chiu, W., Kakadiaris, I.A.: Learningbased segmentation framework for tissue images containing gene expression data. IEEE Transactions on Medical Imaging 26, 728-744 (2007)

7. Kurkure, U., Le, Y., Paragios, N., Carson, J., Ju, T., Kakadiaris, I.: Markov random field-based fitting of a subdivision-based geometric atlas. In: Proc. IEEE (ICCV), Barcelona, Spain, November 6-13, pp. 2540-2547 (2011)

8. Ng, L., Pathak, S.D., Kuan, C., Lau, C., Dong, H., Sodt, A., Dang, C., Avants, B., Yushkevich, P., Gee, J.C., Haynor, D., Lein, E., Jones, A., Hawrylycz, M.: Neuroinformatics for genome-wide 3D gene expression mapping in the mouse Brain. IEEE/ACM Transactions on Computational Biology and Bioinformatics 4(3), 382-393 (2007)

9. Yi, Z., Soatto, S.: Correspondence transfer for the registration of multimodal images. In: Proc. IEEE ICCV, Rio de Janeiro, Brazil, October 14-21, pp. 1-8 (2007)

10. Sotiras, A., Ou, Y., Glocker, B., Davatzikos, C., Paragios, N.: Simultaneous Geometric - Iconic Registration. In: Jiang, T., Navab, N., Pluim, J.P.W., Viergever, M.A. (eds.) MICCAI 2010, Part II. LNCS, vol. 6362, pp. 676-683. Springer, Heidelberg (2010)

11. Kurkure, U., Le, Y.H., Paragios, N., Carson, J.P., Ju, T., Kakadiaris, I.A.: Landmark/image-based deformable registration of gene expression data. In: Proc. IEEE (CVPR), Colorado Springs, CO, June 21-23, pp. 1089-1096 (2011)

12. Maes, F., Collignon, A., Vandermeulen, D., Marchal, G., Suetens, P.: Multimodality image registration by maximization of mutual information. IEEE Transactions on Medical Imaging 16(2), 187-198 (1997)

13. Ou, Y., Davatzikos, C.: DRAMMS: Deformable Registration via Attribute Matching and Mutual-Saliency Weighting. In: Prince, J.L., Pham, D.L., Myers, K.J. (eds.) IPMI 2009. LNCS, vol. 5636, pp. 50-62. Springer, Heidelberg (2009)

14. Carson, J.P., Ju, T., Bello, M., Thaller, C., Warren, J., Kakadiaris, I.A., Chiu, W., Eichele, G.: Automated pipeline for atlas-based annotation of gene expression patterns: Application to postnatal day 7 mouse brain. Methods 50, 85-95 (2010) 